\title{
Architecture of the Hybrid Intelligent Multi-agent System of Heterogeneous Thinking for Planning of Distribution Grid Restoration
}

\author{
Sergey LISTOPAD \\ Kaliningrad Branch of the Federal Research Center "Computer Science and Control" of the \\ Russian Academy of Sciences, Gostinaya Str. 5, Kaliningrad 236022, Russian Federation \\ ser-list-posteyandex.ru
}

\begin{abstract}
The problems of dynamic systems' management, in particular the regional power distribution grid, are characterized by heterogeneity, lack of time for decision-making, distribution and partial observability of the control object, as well as the interdependence of actions performed and decisions made. Traditional abstract mathematical methods used in electric power industry are not relevant to such problems due to their inherent non-factors, and therefore solve only well formalized parts of these problems. To provide information support for solving problems in dynamic environments, a new class of intelligent systems is proposed, which simulate collective decision-making under the guidance of a facilitator, namely hybrid intelligent multi-agent systems of heterogeneous thinking. The presence of a hybrid component in these systems provides the opportunity to work with the heterogeneity of problems, and the presence of intelligent selforganizing agents make it possible to relevantly model effective problem-solving practices of expert teams in order to provide operational dispatching personnel with relevant solutions under time pressure. The paper considers the architecture of such a system for solving the problem of restoring the regional power distribution grid after large-scale accidents.
\end{abstract}

Keywords: heterogeneous thinking, hybrid intelligent multi-agent system, distribution grid restoration

\section{Introduction}

Management of complex dynamic systems, such as power grid, urban transport system, emergency services, is characterized by diversity and significant amounts of continuously flowing heterogeneous information, interdependence of decisions made, variability of the problem environment in real time, influence of the human factor, in particular, incorrect interpretation of data, blindness to changes etc. The lack of time to make decision in such systems causes the incompleteness of the knowledge and data used (Fominykh, 2018).

Traditionally, for operational dispatching of such systems, guidelines and operational procedures are developed for solving typical problems like power supply restoration after large-scale accidents. In power distribution organizations, such instructions are created, in particular, based on the results of an analysis of previous accidents by teams 
of power engineering experts, representatives of design institutes who have developed the generation and power distribution grid facilities of the organization, as well as representatives of equipment manufacturers. However, the emergency conditions of the system can seriously differ from those adopted during the development of the plan, which reduces the effectiveness of remedial measures, leading to unacceptable loads, voltage levels or the operation of protection systems (Barabashi, 2003). At the same time, while decision-making in a dynamic environment, it is not possible to organize detailed collective discussion every time an accident occurs.

In this regard, the development of intelligent information systems that integrate the knowledge of experts of various specialties, the coordination of several optimality criteria and the consideration of a multitude of constraints in a dynamic, directly unobservable environment and lack of time to make a decision are relevant. To model such structures in power distribution organizations, a new class of intelligent systems is proposed, namely hybrid intelligent multi-agent systems of heterogeneous thinking (HIMASHT). They combine hybrid intelligent approach (Kolesnikov, 2001), providing the integration of various experts' knowledge, apparatus of multi-agent systems (Wooldridge and Jennings,1995; Tarasov, 2002; Serugendo et al., 2005), allowing to model collective reasoning of experts, and methods of heterogeneous thinking (Gardner, 1993; De Bono, 1994; Kaner et al., 2011), representing effective collaboration practices in expert teams. The main purpose of HIMASHT is providing accident recovery plan that define switching and recovery actions sequence under time constraints to the operational and dispatching personnel of the energy supplying organization, which would be of comparative quality with that of real expert teams.

\section{The problem of distribution grid restoration after large-scale accident}

In the simplest "game" form, the power grid restoration problem can be described as follows. The power grid is represented by a graph $P S=\langle V, E\rangle$ with three types of

nodes $V$ : power centre $v^{s} \in V^{s} \subseteq V$, consumer $v^{l} \in V^{l} \subseteq V$, and bus $v^{b} \in V^{b} \subseteq V$. The edge $E$ of the graph denote the power line with switch, turning it on or off. Closed lines form a radial structure, i.e. there are no cycles of such lines in the graph. The power centre is described by the magnitude of the maximum generated power, the consumer is characterized by the magnitude of the rated power consumption, and the state (powered / disconnected), and the power lines are described by the state (closed / opened), transmission capacity, and operability (good / accident). It is required to determine which lines need to be closed / opened, and in what order, to ensure the maximum possible amount of power consumption while observing operational limitations: maintaining the radial structure of the closed lines, the total value of loads that are fed through each line should not exceed its transmission capacity.

An example of a power grid graph for such problem is shown in Figure 1. In the left part of the figure in the normal state all the loads are distributed between the two power centres, there are no cycles of powered lines. When an accident occurs in such power grid, three consumers marked with dashed arrows de-energize. The situation of power supply restoration, when all three disconnected consumers cannot be powered from one power line due to overload, is shown in Figure 1 on the right. In this case, de-energized section is divided into two parts by opening the circuit breaker closed in the normal state. 
After that, it becomes possible to power de-energized consumers from different lines of the working part of the grid.

Emergency situation

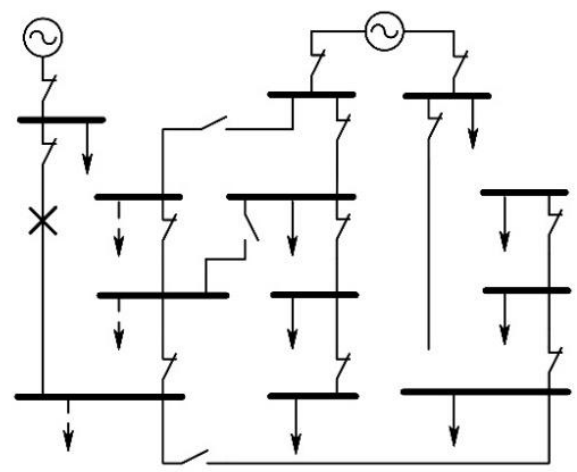

Supply restoration situation

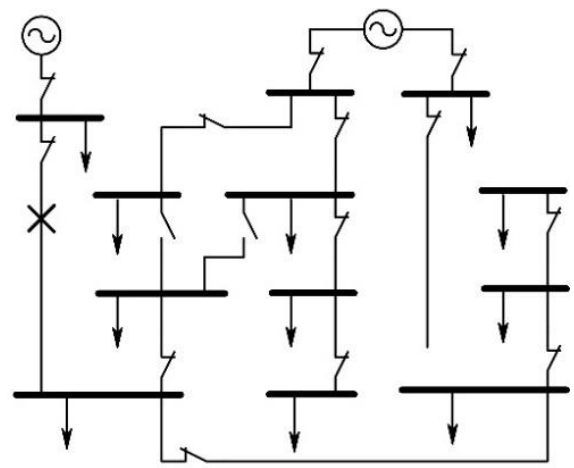

Denotations:

(2) - power center; $\longrightarrow$ - bus; $\longrightarrow \longrightarrow$ - closed line; $\longrightarrow$ - opened line; $\longrightarrow$ - powered consumer;

$\longrightarrow \rightarrow$ - de-energized consumer; $X$ - line failure

Figure 1. An example of the power grid scheme for "game" restoration problem

Such "game" power recovery problem is used to test optimization methods for subsequent coarse- or fine-grained hybridization in order to solve the distribution grid restoration-planning problem (DGRPP). Without hybridization, game planning methods are irrelevant to DGRPP due to a significantly larger number of object types and their properties taken into account for constructing an admissible plan, as well as non-factors inherent to DGRPP in the sense of A.S. Narinyani (Narinyani, 2008). The increase in the number of types of simulated objects is associated, for example, with the impossibility of remote switching in some parts of the power grid, the presence of distributed generation and active consumers, the need to take physical processes in the power grid into account. DGRPP's non-factors include, for example, the following: the undetermined location of the accident during recovery planning; the inaccuracy of the amount of power consumed by each client and generated by each source of the distributed generation; the fuzziness of the restoration time of power grid elements; incorrect operation of emergency mode sensors; incomplete power grid model.

Based on the analysis of the works (Thiebaux and Cordier, 2001; Uspenskiy and Kyzrodev, 2010; Kyzrodev and Uspensky, 2010; Kumar et al., 2010; Hentenryck, 2015) devoted to planning of distribution grid restoration after accidents, DGRPP was formulated, which is in the development of a grid restoration plan consisting of a sequence of switching events, and a sequence of maintenance crews' trips for switching and restoration works. Input data for DGRPP consists of the following sets: 1) elements of the grid; 2) incidence relations between elements of the grid; 3) locations; 4) routes between locations; 5) repair teams; 6) vehicles; 7) resources for restoration of the grid; 8) actions for restoration of the grid. Optimality criteria for the plan: 1) minimizing the time of disconnection of the priority load; 2) maximizing the total recovered load; 3) 
maximizing the reliability of the grid (resistance to subsequent accidents). Restrictions on the plan: 1) preservation of the radial structure of the powered lines of the grid;2) for each line, the total value of loads that are fed from a source of distributed generation through it should not exceed its transmission capacity; 3 ) compliance with the balance of active and reactive power; 4) voltage and frequency values must be within acceptable limits; 5) consumers not affected by the initial outage should not be turned off as a result of switching; 6) repair team with appropriate admission start work, if the necessary resources are available in their vehicle; 7) vehicle capacity; 8) working time of teams; 9) vehicles must return to base; 10) when forcibly dividing the grid to islands, the power lines between the islands must have synchronization equipment for subsequent unification of the islands.

In accordance with A.V. Kolesnikov's problem-structural methodology (Kolesnikov, 2001) the problem is reduced into the following tasks:1) localization of the accident site, taking into account the possibility of sensors' failure, which requires relay protection and automation engineer's knowledge; 2) operational and short-term forecasting of active and reactive power consumed by customers after connecting to the network, for solving which the engineer's in analysing and forecasting energy consumption modes knowledge is required; 3) assessment of requirements for restoration actions of the power grid elements, for solving which power equipment repair engineer's knowledge is relevant; 4) building routes for field crews to perform switching and repairing damaged equipment, which needs distribution grid area head's knowledge; 5) determining the switching order, for solving which the engineer's on power operational modes knowledge is needed; 6) recovery plan development that is the task of coordinating intermediate solutions and integrating private solutions of tasks, for solving which the regional operational and technological dispatcher's knowledge is required.

To solve the DGRPP wholly, it is proposed to model collective decision making by the operating personnel of the energy supplying organization, power engineers, logisticians, and labour protection specialists using the HIMASHT methods.

\section{The functional structure of the hybrid intelligent multi-agent system of heterogeneous thinking}

For computer simulation of heterogeneous thinking methods in expert teams, the functional structure of the HIMASHT in Figure 2 is proposed, which is the hybrid intelligent multi-agent system (Kolesnikov et al., 2011), extended by the agent-facilitator (AF), organizing collective processes, and the corresponding relations between it and the expert agents, each of which is responsible for solving one of DGRPP's tasks mentioned above. Modeling collective heterogeneous thinking suggests that AF initiates various methods of collective interaction of agents, "thinking styles" depending on the current situation. For this purpose, it has to identify the stages of the problem-solving process, the composition of expert agents and their "thinking style", the current situation in HIMASHT, the positive and negative group effects. Also it acts on expert agents activating relevant to the situation "thinking style" to minimize negative effects and to reinforce positive ones, using, among other, the "diamond of participatory decisionmaking" model presented in fig 3 (Kaner et al., 2011). According to this model, the problem solving process in the HIMASHT goes through three stages, which corresponds agents" "thinking style": divergent thinking, groan and convergent thinking. At the stage of divergent thinking, the expert agents generate a variety of the problem's solutions, 
and the AF stimulates their development by appropriate methods, for example brainstorming with leading questions, brain-writing pool, challenging restrictions and lateral thinking. (Kolesnikov and Listopad, 2018). If even with the use of divergent thinking methods there are no contradictions, i.e. the problem has an obvious solution, the process is completed. Otherwise, conflicts arise of between HIMASHT's agents over knowledge, beliefs, opinions, i.e. a kind of cognitive conflicts (Tang and Basheer 2017; Kolesnikov and Listopad, 2018).

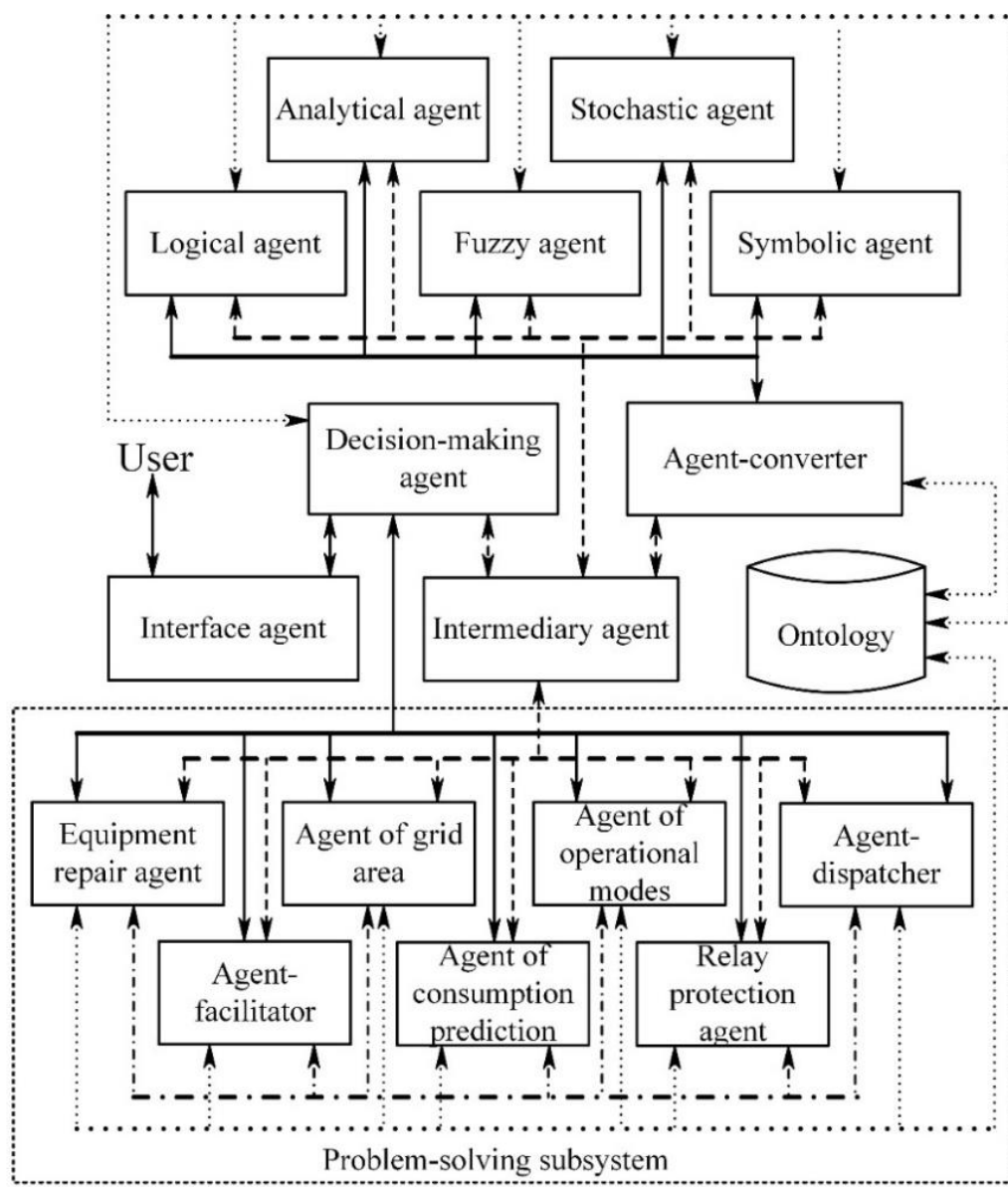

Denotations:

$\longrightarrow$ - agents relations: information requests and responses,

$---\rightarrow$ - agents relations: requests for assistance in solving problems,

........ - interaction of agents with the ontology,

-..- facilitating relations

Figure 2. The functional structure of the hybrid intelligent multi-agent system of heterogeneous thinking 
Conflict is a distinctive feature of the groan stage, allowing the AF to take measures to bring together agents' points of view using such methods as looking from other's point of view, returning from decisions to needs and parallel thinking (Kolesnikov and Listopad, 2018). At the stage of convergent thinking, the agents will jointly reformulate and refine the proposed solutions until they receive a collective decision that is relevant to the diversity of experts' models presented in the HIMASHT consensus, voting and choice of decision-making agent after discussion with expert agents (Kolesnikov and Listopad, 2018). The number of "switching" of thinking methods is not determined in advance, since the groan stage may be absent, and different methods at the divergent and convergent thinking stages can be used consistently.



Denotations: $\odot$ - alternative; $\otimes$ - early uncoordinated solution; $\bigotimes$ - negotiated solution

Figure 3. The "diamond of participatory decision-making" model by Kaner et al.

Other agents presented in Figure 2 are traditional for hybrid intelligent multi-agent system. The interface agent is responsible for user interaction: it requests input data, gives the result, and visualizes the problem solving process. The decision-making agent (DMA) models the work of the head of the central dispatching service of the regional dispatch management. It sends tasks to the expert agents, collects the results of work, determines whether the stopping criterion is reached, and makes the final decision or starts a new iteration of the problem solving process. Expert agents (relay protection agent, agent of consumption prediction, equipment repair agent, agent of grid area, agent of operational modes; agent-dispatcher) having knowledge about the corresponding "specialty" of the subject area and, modelling a certain kind of thinking, generate solutions. The intermediary agent performs work on tracing the names, models and capabilities of registered agents. Intelligent technology agents in the upper part of Figure 2 (analytical, stochastic, logical, fuzzy, symbolic) together with the agent-converter implement the hybrid component of the HIMASHT, combining heterogeneous knowledge, methods, models and algorithms for solving problems. The ontology is the semantic network common for all agents, the basis for agent interaction. It is based on the conceptual model of the problem being solved and describes distribution grid resources (consumers, buses, switches, power lines, repair crews, vehicles and others) their properties and methods for working with them, types of transmitted messages, HIMASHT architecture models etc.

Interaction of agents in HIMASHT is described by protocols (Singh et al., 2014), defining schemes (distributed algorithm) for the exchange of information, knowledge, agent coordination in solving tasks. Agent relations, such as the information requests and responses and requests for assistance in solving problems (Figure 2), are described by the standard protocol of speech acts (Weerasooriya et al., 1994). The interaction of 
agents with the subject area is provided by the software platform on which HIMASHT is implemented by processing requests for creating the objects represented in it and executing the methods defined in these objects. Facilitation relationships are described by a heterogeneous thinking protocol, which is an extended protocol of speech acts (Kolesnikov and Listopad, 2019).

Let us consider in more detail the architecture of the HIMASHT agents.

\section{Agent Architectures}

The interface agent architecture is shown in Figure 4. The HIMASHT message router is not part of the agent, but is a messaging subsystem of the software platform on which the HIMASHT is implemented, providing message delivery between agents. The message receiving/sending subsystem allows the agent to interact with other agents, recording messages in a language understandable to other agents, for example, KQML or ACL. The ontology interpreter provides message generation using the HIMASHT ontology (Figure 2), analyses the semantics of the body of parsed messages from message receiving/sending subsystem, generates program objects on the basis of message body and HIMASHT ontology, and then routes them to the proper subsystem according to the message type and content. The routing algorithm of ontology interpreter is different for different types of agents, but in general, message receiving/sending subsystem and ontology interpreter are typical for the HIMASHT agent, and when considering the architectures of other agents, their descriptions are omitted. The input / output subsystem provides user interaction, requests input data, reports the result, provides the ability to edit the database. The database of DGRPP's objects stores information about the distribution grid resources (consumers, buses, switches, power lines, repair crews, vehicles and others), actions and their properties, determining the conditions of the problem to be solved.

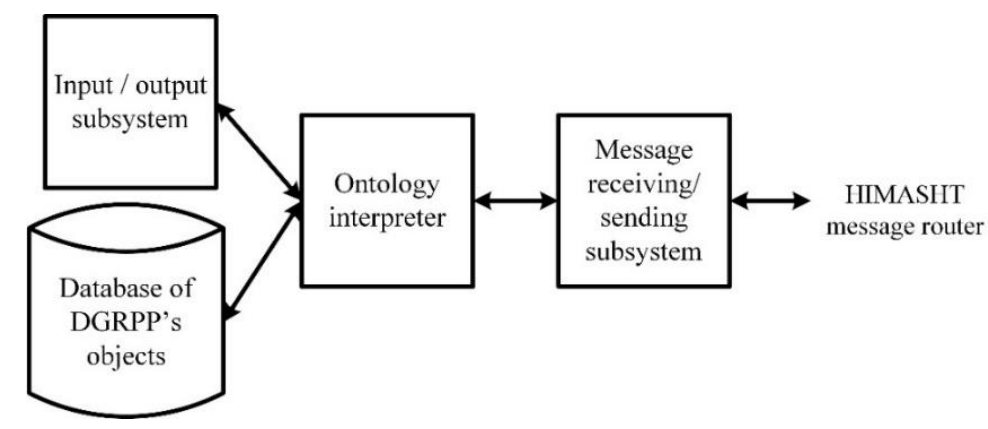

Figure 4. The interface agent architecture

The architecture of the DMA is shown in Figure 5. The problem decomposition subsystem, based on the ontology analysis, distributes among the expert agents the tasks of the DGRPP and the initial data necessary for their solution. DMA's solution evaluating subsystem calculates the index of the quality of solutions submitted by expert agents in accordance with the objective function specified by the developer of 
HIMASHT. If no solutions of satisfactory quality were found, this subsystem launches a new iteration of the problem solving by HIMASHT. The base of decision-making algorithms stores a set of algorithms used by the agent when choosing the final decision depending on the thinking style by the AF. The interpreter of decision-making algorithms is a subsystem for execution of decision-making algorithms.

The architecture of the AF is shown in Figure 6. The subsystem for analysing the situation of collective problem solving performs the identification of the current stage of the problem solving process in accordance with the "diamond of participatory decisionmaking" model (Figure 3). The subsystem for choosing of heterogeneous thinking method using fuzzy knowledge base imitates the work of a facilitator in choosing the "thinking style" of the team that is relevant to the collective problem-solving situation. The fuzzy knowledge base of methods' relevance describes the rules for choosing the "thinking style" of agents depending on the decision-making situations in the HIMASHT, as well as various features of the problems. To form such a base, it is necessary to complete a series of computational experiments and establish a correspondence between the class of problems and the relevant methods of heterogeneous thinking.

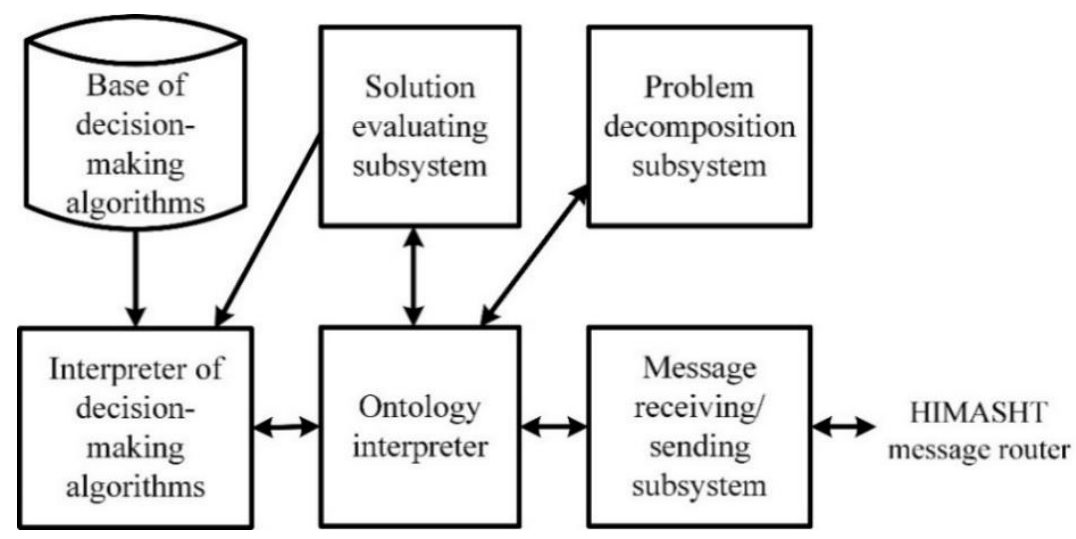

Figure 5. The architecture of the decision-making agent

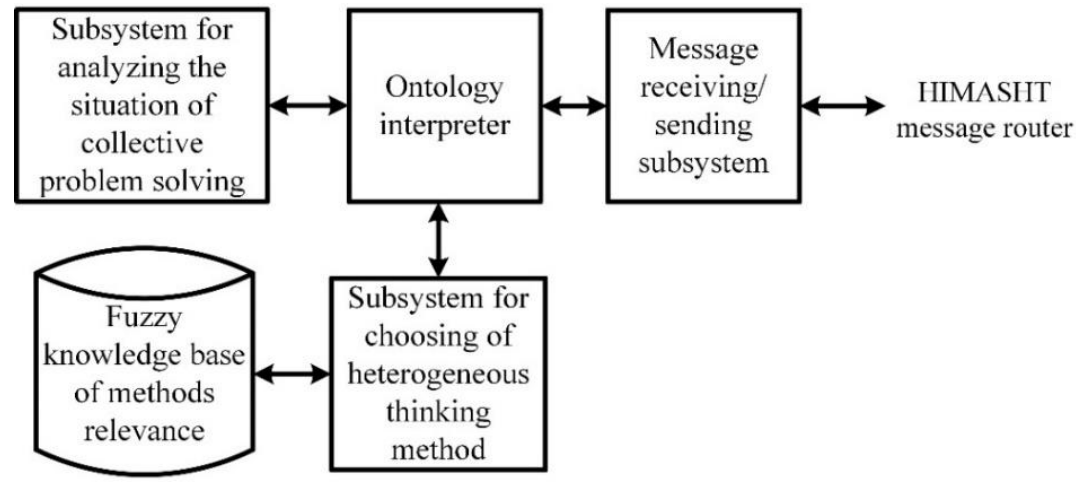

Figure 6. The architecture of the agent-facilitator 
The architecture of the intelligent technology agent is shown in Figure 7. Algorithms of intelligent technology implement the corresponding problem-solving methods: algebraic equations; Monte Carlo method; production expert system; Mamdani fuzzy inference algorithm etc. It solves tasks transmitted by expert agents and other intelligent technology agents.

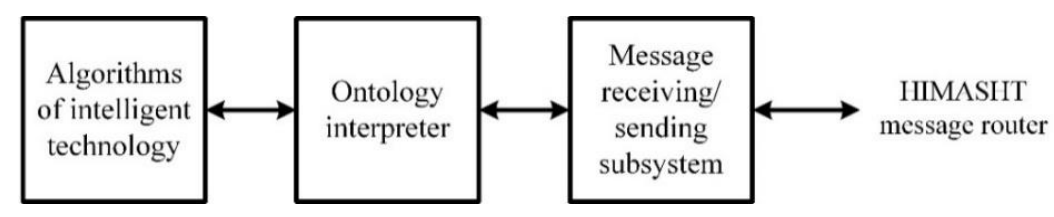

Figure 7. The architecture of the intelligent technology agent

The architecture of the expert agent is shown in Figure 8. The base of heterogeneous thinking algorithms stores a set of algorithms that the expert agent performs with the interpreter when solving its task, depending on the "thinking style" set by the AF, taking into account its goal. They may contain requests for intelligent technology agents to perform certain functions. The goal adjustment algorithm allows adjusting agent's goal function in consequence of interaction with other agents at the groan stage. The belief base contains the agent's beliefs regarding the ontology, which can be supplemented and corrected by the belief correction algorithm in consequence of interaction with other agents and the domain model at the groan stage.

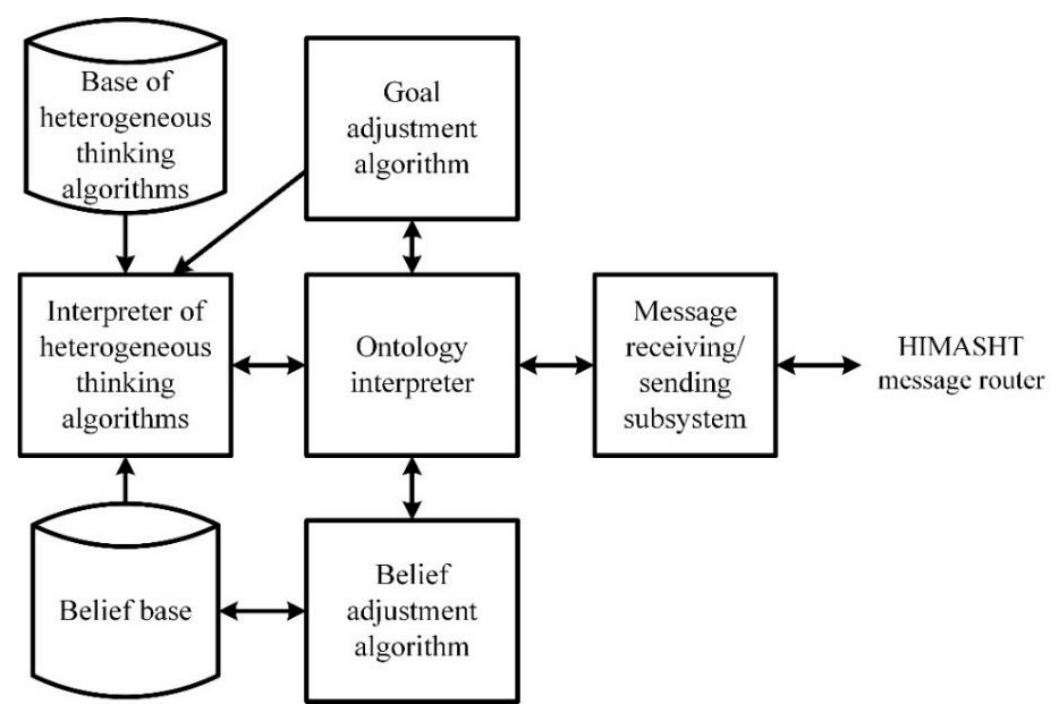

Figure 8. The architecture of the expert agent

The architecture of the intermediary agent is presented in Figure 9. The database management subsystem adds, deletes, modifies and searches for agent records in the 
database (list) of agents. The agent database stores in the form of records information about registered agents and their capabilities.

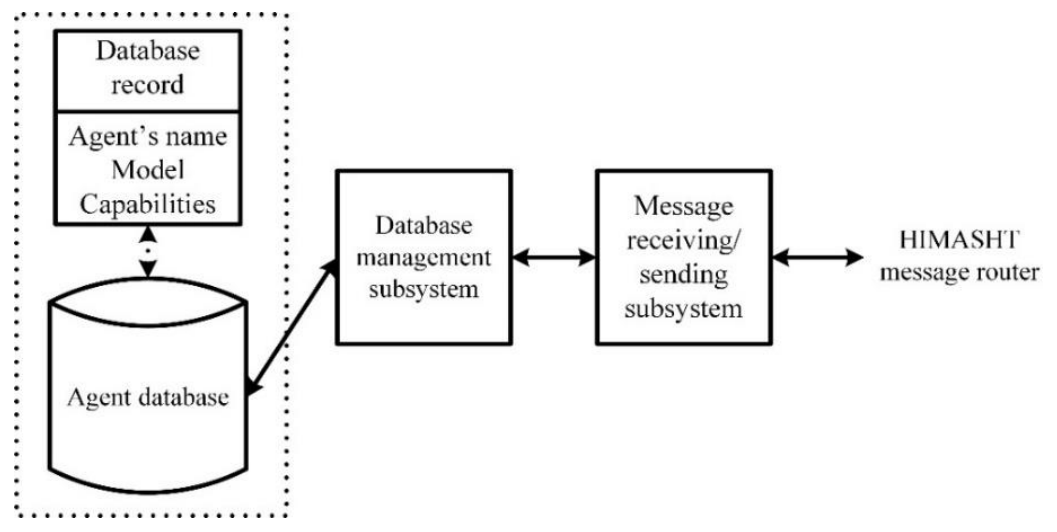

Figure 9. The architecture of the intermediary agent

As can be seen from the analysis of agent architectures in Figures 4 - 9, they are mainly reactive agents that perform functions specified by the developer in response to incoming messages from other agents or to user actions. The exception is expert agents who have dynamic models of goal setting and beliefs. Initially, the developers of the system set expert agents' goals and beliefs; however, when working on problems at the groan stage, they exchange data and knowledge substantiating the proposed solutions to the problem, and, if necessary, modify their goals and beliefs. Thus, after solving a certain number of problems, the goals and beliefs of expert agents may differ from those set during development. Having the opportunity to request assistance in solving a problem from any of the intelligent technology agents, expert agents each time solving a new problem form a new integrated (hybrid) solution method relevant to it, demonstrating signs of "strong" self-organization, that is, arising due to the distributed interaction of agents without explicit centralized management (Serugendo et al., 2005).

\section{System's effectiveness estimation}

To evaluate accurately the effectiveness of the proposed HIMASHT architecture, it is necessary to accomplish its software implementation and conduct a series of computational experiments with various models of power grids. At the moment, a rough estimate of the HIMASHT effectiveness can be given by comparing its capabilities with other implemented systems designed to solve problems in various areas of the economy. For comparative analysis, two intelligent system is used: 1) hybrid intelligent system AGRO (Kolesnikov, 2001) for crop forecasting and planning of agricultural events, which allowed to increase the planning quality by $7-14 \%$, and the planning speed by four times; 2) hybrid multi-agent intelligent system TRANSMAR (Kolesnikov et al., 2014), designed to solve complex transport and logistics problems and provided an increase in the efficiency of routing by more than $7 \%$, and routing speed by $23 \%$ compared to methods existed at the moment of its creation. 
Table 1. Comparative analysis of the features of intelligent systems for solving heterogeneous problems

\begin{tabular}{|l|c|c|c|}
\hline \multicolumn{1}{|c|}{ Features } & AGRO & TRANSMAR & HIMASHT \\
\hline Handling problem heterogeneity & + & + & + \\
\hline Handling tool heterogeneity & + & + & + \\
\hline Modelling expert reasoning & + & - & + \\
\hline Autonomy of elements / agents & - & + & + \\
\hline Ontology-based reasoning & - & + & + \\
\hline $\begin{array}{l}\text { Modelling collective heterogeneous } \\
\text { thinking }\end{array}$ & - & - & + \\
\hline Self-organization type & - & Weak & Strong \\
\hline
\end{tabular}

Designations: + - feature present; - - no feature

As shown in Table 1 the proposed class of HIMASHT combines the representation of the heterogeneous functional structure of the problem with heterogeneous structure of the expert team and heterogeneous collective thinking methods, creating conditions for solving problems in dynamic environments without simplification and idealization. Due to the implementation of the system's heterogeneous elements as autonomous intelligent agents and ontology-based reasoning, the HIMASHT can effectively adapt to changing conditions of the problem, including modifying its structure and parameters, and develop a new relevant method during each problem solving process, showing signs of "strong" self-organization. Thus, HIMASHT has advantages over AGRO and TRANSMAR and more relevant to real expert teams solving problems in dynamic environments, therefore, as result of its software implementation, performance indicators could be no worse than those of reviewed intelligent systems could.

\section{Conclusion}

The paper discusses the features of complex dynamic systems managing by the example of the DGRPP. The functional structure of the HIMASHT is presented, as well as the architecture of its agents implementing heterogeneous thinking methods for the relevant modelling of the problem solving process by expert team. The proposed HIMASHT moves the imitation of the collective development of operational actions to the field of synergetic informatics, when interaction between the elements of an intelligent system is no less important than their composition and quantity. This leads to self-organizing, social management models, each element of which is developing, obtaining data and knowledge from other elements. This reduces the cost of developing and operating the system. Modelling the methods of heterogeneous thinking by the agents of the system allows it to adapt to the dynamically changing conditions of the problems, each time reestablish connections between the agents, choosing the interaction style and developing a new decision-making method relevant to the situation. The use of such systems in operational dispatching management will make it possible to develop solutions relevant to the problems that arise in the complex, dynamic environments of regional power grids. 


\section{Acknowledgements}

The reported study was funded by RFBR according to the research project №18-0700448A.

\section{References}

Barabashi, A.L., Bonabo, E. (2003) Bezmasshtabnyye seti [Non-scale networks]. V mire nauki [In the world of science], 8:54-63. (in Russian)

De Bono, E. (1994) Parallel Thinking: From Socratic to De Bono Thinking, Penguin Books, Melbourne.

Fominykh, I.B., Pozhidayev, A.K., Romanchuk, S.V. (2018) Imitatsiya rassuzhdeniy $v$ intellektual'nykh sistemakh myagkogo i zhestkogo real'nogo vremeni [Imitation of reasoning in intellectual systems of soft and hard real time].In: Gibridnyye i sinergeticheskiye intellektual'nyye sistemy: Materialy IV Vserossiyskoy Pospelovskoy konferentsii s mezhdunarodnym uchastiyem [Hybrid and synergetic intelligent systems: Proceedings of the IV All-Russian Pospelov conference with international participation], p. 69-79. (in Russian).

Gardner, H. (1993) Multiple Intelligences - The Theory in Practice, Basic Books, New York.

Hentenryck, P., Coffrin C. (2015) Transmission system repair and restoration. Mathematical Programming. Ser. B, 151:347-373.

Kaner, S., Lind, L., Toldi, C., Fisk, S., Beger, D. (2011) The Facilitator's Guide to Participatory Decision-Making, Jossey-Bass, San Francisco, CA.

Kolesnikov, A.V. (2001) Gibridnye intellektual'nye sistemy. Teoriya i tekhnologiya razrabotki [Hybrid intelligent systems: theory and technology of development], Izdatel'stvo SPbGTU, Saint Petersburg. (in Russian).

Kolesnikov, A.V., I.A. Kirikov, and S.V. Listopad (2014) Gibridnye intellektual'nye sistemy s samoorganizatsiey: koordinatsiya, soglasovannost', spor [Hybrid intelligent systems with self-organization: coordination, consistency, dispute], IPI RAN, Moscow. (in Russian).

Kolesnikov, A.V., Kirikov, I.A., Listopad, S.V., Rumovskaya, S.B., Domanitskiy, A.A. (2011) Reshenie slozhnykh zadach kommivoyazhera metodami funktsional'nykh gibridnykh intellektual'nykh system [Complex travelling salesman problems solving by the methods of the functional hybrid intelligent systems], IPI RAN, Moscow. (in Russian).

Kolesnikov, A.V., Listopad, S.V. (2018) Model' gibridnoy intellektual'noy mnogoagentnoy sistemy geterogennogo myshleniya dlya informatsionnoy podgotovki operativnykh resheniy $v$ regional'nykh elektricheskikh setyakh [Model of a hybrid intelligent multiagent system of heterogeneous thinking for preparation of information about operational decisions in a regional power system]. Sistemy i sredstva informatiki [Systems and Means of Informatics], 28(4):31-41. (in Russian).

Kolesnikov, A.V., Listopad, S.V. (2019) Protokol geterogennogo myshleniya gibridnoy intellektual'noy mnogoagentnoy sistemy dlya resheniya problemy vosstanovleniya raspredelitel'noy elektroseti [Heterogeneous thinking protocol of hybrid intelligent multiagent system for solving distributional power grid recovery problem]. Informatika i yeye primeneniya [Informatics and applications], 13(2):76-82. (in Russian).

Kumar, V., Kumar, H.C.R., Gupta, I., Gupta, H.O. (2010) DG integrated approach for service restoration under cold load pickup. IEEE Transactions on Power Delivery, 25(1):398-406.

Kyzrodev, I.V., Uspensky M.I. (2010) Avtomatizatsiya vosstanovleniya elektrosnabzheniya $v$ raspredelitel'nykh setyakh [Automating the restoration of power supply in distribution networks]. Izvestiya Komi nauchnogo tsentra UrO RAN [News of the Komi Scientific Center, Ural Branch of the Russian Academy of Sciences], 2:84-91. (in Russian).

Narinyani A.S. (2008) Inzheneriya znaniy i NE-faktory: kratkiy obzor-08 [Knowledge Engineering and Non-Factors: A Brief Overview-08]. Voprosy iskusstvennogo intellekta [Artificial Intelligence Issues], 1:61-77. (in Russian). 
Serugendo, G.D.M., Gleizes M.-P., Karageorgos A. (2005) Self-organization in multiagent systems. The Knowledge engineering review, 20(2):165-189.

Singh, R., Singh, A., Mukherjee, S. (2014) A Critical Investigation of Agent Interaction Protocols in Multiagent Systems. International Journal of Advancements in Technology, 5(2):72-81.

Tang, A.Y.C., Basheer, G.S. (2017) A Conflict Resolution Strategy Selection Method (ConfRSSM) in Multi-Agent Systems. International Journal of Advanced Computer Science and Applications, 8(5):398-404.

Tarasov, V.B. (2002) Ot mnogoagentnykh sistem $k$ intellektualnym organizatsiyam: filosofiya, psikhologiya, informatika [From multiagent systems to intelligent organizations: philosophy, psychology, and informatics], Editorial URSS, Moscow. (in Russian).

Thiebaux, S., Cordier, M.-O. (2001) Supply Restoration in Power Distribution Systems - A Benchmark for Planning under Uncertainty. In: Proceedings of the 6th European Conference on Planning (ECP-01), p. 85-96.

Uspenskiy, M.I., Kyzrodev, I.V. (2010) Metody vosstanovleniya elektro-snabzheniya $v$ raspredelitel'nykh setyakh [Methods for restoring electrical supply in distribution networks], Syktyvkar. (in Russian).

Weerasooriya, D., Rao, A.S., Ramamohanarao, K. (1994) Design of a concurrent agent-oriented language. Lecture Notes in Computer Science, 890:386-401.

Wooldridge, M., Jennings, N. (1995) Agent Theories, Architectures and Languages: a Survey. In: Intelligent Agents: ECAI-94 Workshop on Agent Theories, Architectures and Languages, p. $1-22$.

Received November 18, 2019, accepted November 19, 2019 